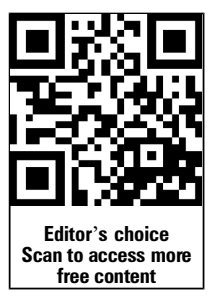

\title{
Size and anatomic location of ruptured intracranial aneurysms in patients with single and multiple aneurysms: a retrospective study from a single center
}

\author{
Bharathi Dasan Jagadeesan, ${ }^{1,2}$ Josser E Delgado Almandoz, ${ }^{3}$ Yasha Kadkhodayan, ${ }^{3}$ \\ Colin P Derdeyn, ${ }^{4,5,6}$ Dewitte T Cross III, ${ }^{4,5}$ Michael R Chicoine, ${ }^{5}$ Keith M Rich, ${ }^{4,5,7}$ \\ Gregory J Zipfel, ${ }^{5,6}$ Ralph G Dacey, ${ }^{5}$ Christopher J Moran ${ }^{4,5}$
}

${ }^{1}$ Department of Radiology, University of Minnesota, Minneapolis, Minnesota, USA ${ }^{2}$ Department of Neurosurgery, University of Minnesota, Minneapolis, Minnesota, USA ${ }^{3}$ Division of Interventional Neuroradiology, Abbott Northwestern Hospital, Minneapolis, Minnesota, USA ${ }^{4}$ Department of Radiology, Washington University School of Medicine, St Louis, Missouri, USA

${ }^{5}$ Department of Neurological Surgery, Washington University School of Medicine, St Louis, Missouri, USA

${ }^{6}$ Departments of Neurology, Washington University School of Medicine, St Louis, Missouri, USA

${ }^{7}$ Department of Neurobiology, Washington University School of Medicine, St Louis, Missouri, USA

\section{Correspondence to}

Dr Bharathi Dasan Jagadeesan, Department of Radiology, University of Minnesota, MMC 292 Mayo Memorial Building, 420 Delaware St SE

Minneapolis, MN 55455, USA; jagad002@umn.edu

Received 15 December 2012 Accepted 6 March 2013 Published Online First 28 March 2013

\section{ABSTRACT}

Background and purpose The difference in the relationship between the size of intracranial aneurysms (IAs) and their risk of rupture in patients with singe IAs versus those with multiple IAs is unclear. We sought to retrospectively analyze the size of ruptured IAs (RIAs) in patients with single and multiple IAs in order to study this relationship further.

Methods We retrospectively measured the size and location of RIAs in all patients who presented to our institute with an acute subarachnoid hemorrhage between 1 January 2005 and 31 December 2010. The IAs were classified by size into very small IAs or VSAs $(\leq 3 \mathrm{~mm})$, small IAs or SAs (>3 mm but $\leq 7 \mathrm{~mm})$ and others (>7 mm).

Results 379 patients (281 with a single IA, Group 1 and 98 with multiple IAs, Group 2) with 419 treated RIAs were included in the study. VSAs and SAs constituted the majority of RIAs in both groups (33.5\% and $45.2 \%$ in Group 1 and $24.6 \%$ and $50.7 \%$ in Group 2) and the mean size of the RIAs was not different between the two groups. VSAs constituted almost two-thirds of all RIAs in certain locations whereas IAs $>7 \mathrm{~mm}$ in size did not constitute more than a third of the RIAs at any of the arterial locations.

Conclusions The high incidence of VSAs, particularly in certain locations in both patient subgroups, suggests that current diagnostic, prognostic and therapeutic options in the management of IAs should be more tailored towards the management of these difficult-totreat lesions.

\section{INTRODUCTION}

Subarachnoid hemorrhage (SAH) from ruptured intracranial arterial aneurysms is a devastating condition. ${ }^{1}$ Various attempts have been made to identify risk factors for aneurysmal rupture, of which the size of the aneurysm remains the most widely studied. However, the relationship between the size of intracranial arterial aneurysms and their risk of rupture remains controversial. Although the data from the prospectively designed International Study of Unruptured Intracranial Aneurysms (ISUIA) trial $^{2}$ suggested that aneurysms which measure $<7 \mathrm{~mm}$ in size have a very low risk of rupture, a number of large case series have since been published which show that the majority of ruptured intracranial aneurysms are $<10 \mathrm{~mm}$ in size. $^{3-6}$ We report the size and distribution of ruptured intracranial arterial aneurysms in a consecutive series of 379 patients with single and multiple aneurysms evaluated by digital subtraction angiography (DSA) at our hospital following presentation with acute SAH during a 5-year period from January 2005 to December 2010.

\section{MATERIALS AND METHODS}

We retrospectively reviewed our prospectively maintained database of DSA studies on patients who presented to our institution between 2005 and 2010 with SAH for the size and distribution of ruptured saccular aneurysms. This included patients who presented with an acute SAH on non-contrast head CT as well as patients who presented with a sudden severe headache and cerebrospinal fluid xanthochromia. These patients had been evaluated with an initial DSA of the intracranial vessels (which is the standard of care at our institution except in a very small number of patients who are extremely unstable). If the initial DSA study was negative, the patients were subjected to a repeat DSA study approximately 1 week later. When the initial or repeat DSA studies identified an intracranial aneurysm(s), the patients underwent endovascular neurosurgical management or surgical clipping of the aneurysms. Endovascular neurosurgical management consisted of coil embolization of aneurysms or, very rarely, placement of intraarterial stents (for small blister aneurysms). Aneurysm clipping was carried out with open craniotomy, direct visualization of the aneurysm and application of one or more clips at the neck of the aneurysm.

The mode of management in each patient was decided by a group of experienced neurosurgeons and endovascular surgical neuroradiologists. In patients in whom only a single aneurysm was identified, the management decision was based on location and size of the aneurysm, anatomy of the parent artery, age of the patient, severity of clinical presentation and the presence of comorbidities. In patients with multiple aneurysms, if it was possible to identify the ruptured aneurysm based on the 
blood distribution on the CT scans or aneurysm morphology (irregularity of surface or small apical blister on DSA), only this aneurysm was treated with clipping or coiling. In other patients with multiple aneurysms in whom it was difficult to pinpoint the ruptured aneurysm due to either diffuse SAH or close anatomical proximity of aneurysms, even when the pattern of bleeding was not diffuse, more than one aneurysm was treated. In some of these patients with multiple aneurysms, both aneurysmal clipping and endovascular treatment were necessary for the optimal management of the different aneurysms. All patients then had a cerebral angiogram at the end of the treatment to document the results of the treatment. Patients treated by an endovascular approach had diagnostic sequences performed at the end of treatment, while patients treated by a surgical approach underwent an intraoperative angiogram.

The size of the treated aneurysms was determined from the maximal dimensions of the aneurysm measured on the initial DSA study. The measurements were performed on twodimensional images obtained on Siemens Axiom Artis equipment using 'quantitative autocalibration' and electronic calipers, measuring the maximum diameter based on at least four projections. Untreated aneurysms in patients with multiple aneurysms were also measured in a similar fashion. The aneurysms were classified into those $\leq 7 \mathrm{~mm}$ and those $>7 \mathrm{~mm}$ in size; this classification was performed keeping in mind the International Study of Unruptured Intracranial Aneurysms (ISUIA) data. Aneurysms measuring $\leq 7 \mathrm{~mm}$ were then further subclassified as very small aneurysms (VSAs) $(\leq 3 \mathrm{~mm})$ and small aneurysms (SAs) $(>3 \mathrm{~mm}$ but $\leq 7 \mathrm{~mm}$ ).

\section{RESULTS}

Patient subgroups and modalities of treatment

A total of 379 consecutive patients (284 women, $75.1 \%$ ) aged $51.1 \pm 13.1$ years were identified as having undergone emergency endovascular neurosurgical management or aneurysmal clipping for ruptured saccular intracranial aneurysms between 1 January 2005 and 31 December 2010 at our institution. Only one aneurysm was found on DSA studies in 281 of the 379 patients (Group 1). Of these 281 aneurysms, 173 (61.6\%) were coiled and $108(38.4 \%)$ were surgically clipped. The remaining 98 patients had multiple aneurysms (Group 2) and a total of 261 aneurysms were found in this group (approximately 2.66 aneurysms per patient). In 34 of these 98 patients (35\%), more than one aneurysm had to be treated in an emergency manner resulting in treatment of $138(53.1 \%)$ of the total 261 aneurysms. Of these 138 aneurysms, 78 (55.6\%) were treated by coiling, 55 were clipped $(40.6 \%)$ and $5(3.8 \%)$ were treated with endovascular stent placement in the parent vessel.

\section{Aneurysm characteristics}

Overall, a total of 419 aneurysms with a mean size of 5.7 $\pm 3.8 \mathrm{~mm}$ were treated in the 379 patients included in the study. Most of the treated aneurysms $(77.6 \%)$ measured $\leq 7 \mathrm{~mm}$ in size; of these, $30.6 \%$ were VSAs and $47.0 \%$ was SAs (table 1 and figure 1).

In Group 1 patients the mean size of the ruptured aneurysms was $5.8 \pm 4.0 \mathrm{~mm}$ whereas, in Group 2 patients, the mean size of the treated aneurysms was $5.9 \pm 3.4 \mathrm{~mm}$. There was no significant difference in the size of the ruptured aneurysm between these two groups. Aneurysms $>7 \mathrm{~mm}$ did not constitute more than one-third of all ruptured aneurysms at any given location. Furthermore, in some locations such as the pericallosal artery and posterior inferior cerebellar artery (PICA), VSAs constituted almost two-thirds of all ruptured aneurysms (table 2, figure 2A,B).
Table 1 Incidence of very small aneurysms (VSAs, $\leq 3 \mathrm{~mm}$ ) and small aneurysms (SAs, $>3 \mathrm{~mm}$ and $\leq 7 \mathrm{~mm}$ ) in each group of aneurysms

\begin{tabular}{llllll}
\hline \multirow{2}{*}{ Size } & \multirow{2}{*}{ All patients } & \multirow{2}{*}{ Group 1 } & \multicolumn{4}{l}{ Group 2 } \\
\cline { 4 - 6 } & & All & All & Treated & Untreated \\
\hline VSAs (\%) & 30.5 & 33.5 & 47.3 & 24.6 & 73.0 \\
SAs (\%) & 47.1 & 45.2 & 38.8 & 50.7 & 25.4 \\
$>7$ mm (\%) & 22.4 & 21.3 & 13.9 & 24.7 & 1.6 \\
\hline
\end{tabular}

In Group 2 patients the treated (presumed ruptured) aneurysms were in general larger than the untreated (presumed unruptured) aneurysms, which had a mean size of $2.9 \pm 2.0 \mathrm{~mm}$ $(p<0.001)$, and this was found to be true at all vascular beds (figure 3). However, despite this trend, we found that in 9 of the 98 patients in Group 2 (9.2\%) the presumed ruptured aneurysm (which was treated) was not the largest aneurysm.

The most common location for a ruptured aneurysm in the entire patient population was the anterior communicating artery $(29.8 \%)$, followed by the posterior communicating artery (18.2\%), the internal carotid artery (which includes ophthalmic, anterior choroidal, superior hypophyseal, terminal internal carotid artery and other intradural paraclinoid aneurysms $(16.9 \%))$, the middle cerebral artery $(12.1 \%)$, basilar artery (7.5\%), pericallosal arteries (5.6\%) and the PICAs (4.6\%) (table 3 and figure 4). The average size of the ruptured aneurysms at each site is shown in table 3 and figure 5 .

\section{DISCUSSION}

This retrospective analysis shows that almost one-third of ruptured aneurysms are VSAs measuring $<3 \mathrm{~mm}$. This finding has prognostic, diagnostic and management implications. With regard to prognosis, it is important to state that similar findings have also been reported by others ${ }^{3-6}$ who found that most ruptured aneurysms are $<7 \mathrm{~mm}$ although they did not specifically address the incidence of VSAs. It is also important to reconcile these findings with those from prospective follow-up studies of unruptured intracranial arterial aneurysms ${ }^{2} 7$ which showed that aneurysms $<7 \mathrm{~mm}$ (particularly in the anterior circulation) have a very low annual incidence of rupture. Some have argued that this discrepancy between retrospective studies of ruptured aneurysms and prospective studies of unruptured aneurysms probably reflects the fact that aneurysms decrease in size

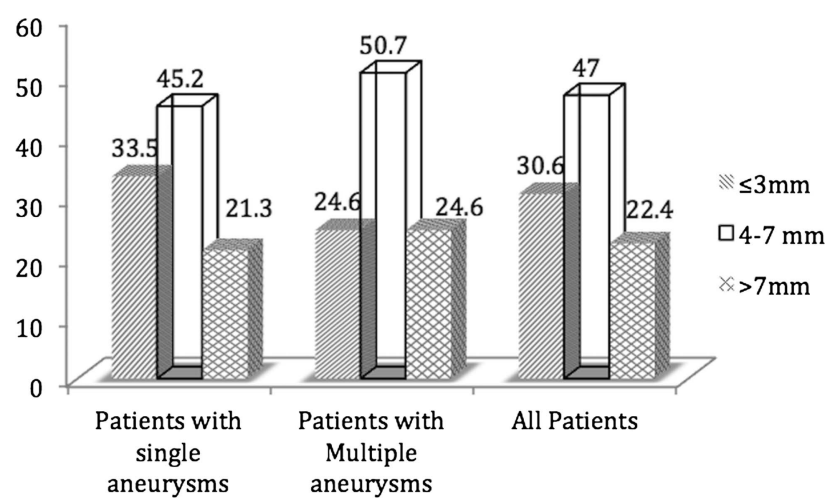

Figure 1 Relative incidence of ruptured (treated) very small aneurysms, small aneurysms and other aneurysms in patients with single aneurysms, patients with multiple aneurysms and in all patients. 
Table 2 Percentage relative incidence of very small aneurysms (VSAs), small aneurysms (SAs) and other ruptured aneurysms at each location

\begin{tabular}{llll}
\hline Location & VSAs & SAs & Others \\
\hline ACOM & 36.7 & 48.4 & 14.9 \\
PCOM & 13.5 & 58.2 & 28.3 \\
ICA & 30.9 & 35.3 & 33.8 \\
MCA & 18.4 & 51.0 & 30.6 \\
Basilar & 29.4 & 44.1 & 26.5 \\
Pericallosal & 62.5 & 25.0 & 22.5 \\
PICA & 66.7 & 33.3 & 0.0 \\
Others & 35.0 & 50.0 & 15.0 \\
\hline
\end{tabular}

ACOM, anterior communicating artery; ICA, internal carotid artery; MCA, middle cerebral artery; PCOM, posterior communicating artery; PICA, posterior inferior cerebellar artery. following a rupture. ${ }^{8} 9$ However, the few reports on aneurysms during the perirupture period show that aneurysms may increase in size immediately prior to a rupture. ${ }^{7}{ }^{10}$ It is therefore more likely that this apparent contradiction reflects the presence of distinct subsets of intracranial aneurysms as suggested by others $^{9} 11{ }^{12}$ - that is, a smaller subset of intracranial aneurysms that grow rapidly and rupture at a smaller size (subset 1) and a larger subset of asymptomatic aneurysms that were included in the prospective trials such as the ISUIA or SUAVe which are at a low risk of rupture (subset 2 ).

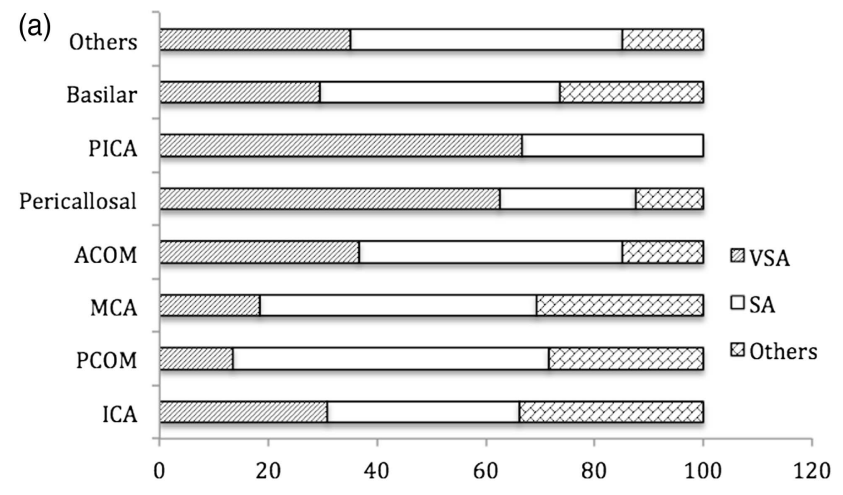

(b)

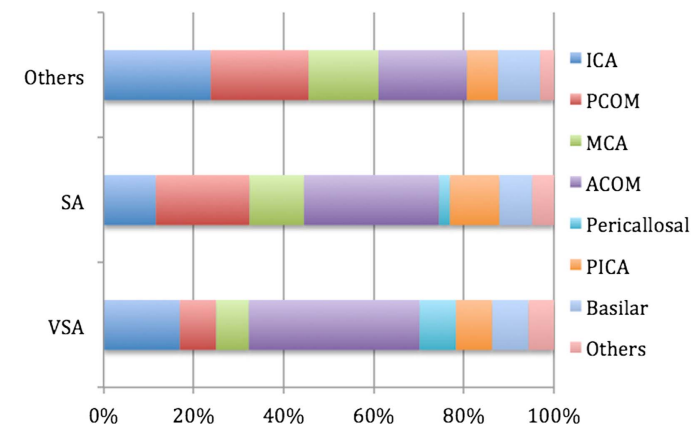

Figure 2 (A) Incidence of very small aneurysms (VSAs), small aneurysms (SAs) and other aneurysms at each anatomical site as a percentage of the total number of ruptured aneurysms at each site. (B) Distribution of VSAs and SAs and other aneurysms in the circle of Willis. ACOM, anterior communicating artery; ICA, internal carotid artery; MCA, middle cerebral artery; PCOM, posterior communicating artery; PICA, posterior inferior cerebellar artery.

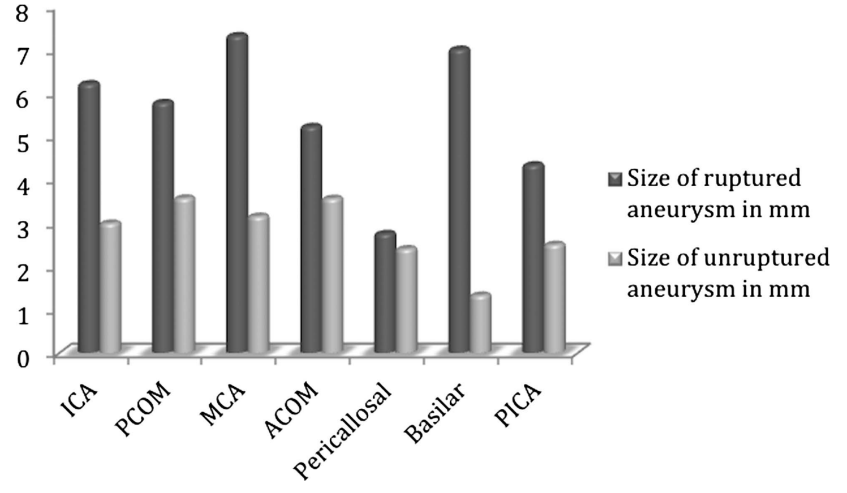

Figure 3 Discrepancy in size (in $\mathrm{mm}$ ) between ruptured and unruptured aneurysms in patients with multiple aneurysms and subarachnoid hemorrhage. ACOM, anterior communicating artery; ICA, internal carotid artery; MCA, middle cerebral artery; PCOM, posterior communicating artery; PICA, posterior inferior cerebellar artery.

Our study suggests that the overall size threshold for aneurysm rupture in aneurysms belonging to subset 1 is likely to be far less than $7 \mathrm{~mm}$. Our study also suggests that this threshold is likely to vary by site, as can be seen from the considerable variation in the relative incidence of SAs and VSAs at each location. Interestingly, the variation in this threshold by location seems to differ from the variation in risk for the rupture of aneurysms by location, which was reported by the ISUIA study. This difference between the populations of ruptured and unruptured aneurysms can also be taken to be an indicator for the existence of distinct subsets of intracranial arterial aneurysms (ie, subsets 1 and 2 described above). Thus, our findings suggest that data arrived at from the ISUIA study alone cannot be applied to prognosticate on the likely outcome in patients with incidentally discovered aneurysms, given that it is currently impossible to distinguish between aneurysms belonging to subset 1 and those belonging to subset 2 based on size and location alone. Factors such as prior SAH, female sex, history of smoking, hypertension and a family history of $\mathrm{SAH}^{13-18}$ must also be considered when providers attempt to offer patients with prognostic information.

With regard to the diagnostic investigation of patients with $\mathrm{SAH}$, our finding that VSAs constitute almost one-third (30.5\%) of all the ruptured aneurysms studied suggests that an initial diagnostic study with very high accuracy for even VSAs needs to be used. Although this role was traditionally performed by DSA studies, in recent years CT angiography (CTA) has emerged as an alternative to the traditional DSA technique for this purpose and several retrospective series have been published which claim that CTA has a high degree of accuracy and should be used as the initial screening study in patients with SAH. ${ }^{19-22}$ However, even 64-slice CTA may have a poor sensitivity for the detection of VSAs, ${ }^{23-25}$ particularly in the setting of diffuse SAH, ${ }^{26}$ and sensitivity is likely to be even poorer when overnight CTA studies are interpreted only by on-call residents. ${ }^{27}$ It may therefore be prudent at present to evaluate patients with SAH with DSA studies including three-dimensional DSA runs.

With regard to the management of patients with SAH, our finding of a high incidence of VSAs is very pertinent given that it is well known that endovascular and surgical treatment of VSAs is harder and associated with a higher rate of complications. $^{28-30}$ The management of patients with SAH is therefore perhaps better performed in high-volume centers with experienced physicians adept at treating VSAs, and recent studies by others showing better outcomes for patients with SAH managed 
Table 3 Incidence and mean size of all ruptured intracranial aneurysms ( $\mathrm{mm})$

\begin{tabular}{lllllll}
\hline $\begin{array}{l}\text { Location of ruptured } \\
\text { aneurysm }\end{array}$ & $\begin{array}{l}\text { All aneurysms } \\
\text { (incidence) (\%) }\end{array}$ & $\begin{array}{l}\text { All patients } \\
\text { (mean size) }\end{array}$ & $\begin{array}{l}\text { Group 1 } \\
\text { (incidence) (\%) }\end{array}$ & $\begin{array}{l}\text { Group 1 } \\
\text { (mean size) }\end{array}$ & $\begin{array}{l}\text { Group 2 } \\
\text { (incidence) (\%) }\end{array}$ & $\begin{array}{l}\text { Group 2 } \\
\text { (mean size) }\end{array}$ \\
\hline ACOM & 29.8 & $4.6 \pm 2.5$ & 33.9 & $4.5 \pm 3.6$ & 20.4 & $5.2 \pm 2.3$ \\
PCOM & 18.2 & $6.4 \pm 3.0$ & 18.2 & $6.6 \pm 4.3$ & 7.1 & $5.8 \pm 2.8$ \\
ICA & 16.9 & $6.6 \pm 5.3$ & 12.2 & $7 \pm 8.1$ & 27.6 & 15.0 \\
MCA & 12.1 & $7.5 \pm 3.3$ & 10.8 & $6.7 \pm 6.8$ & 4.7 & 3.7 \\
Basilar & 7.5 & $6.8 \pm 3.1$ & 8.7 & $5.6 \pm 7.7$ & 3.7 & 7.3 \\
Pericallosal & 5.6 & $5.1 \pm 4.5$ & 6.6 & $3.8 \pm 2.4$ & 2.4 & 8.3 \\
PICA & 4.6 & $3.8 \pm 1.5$ & 5.6 & & & 8.7 \\
Others & 5.3 & $5.5 \pm 3.3$ & 4 & & \\
\hline
\end{tabular}

ACOM, anterior communicating artery; ICA, internal carotid artery; MCA, middle cerebral artery; PCOM, posterior communicating artery; PICA, posterior inferior cerebellar artery.

at high-volume centers perhaps reflect the implications of our results. ${ }^{31-33}$ With regard to specific techniques for the management of these patients, the high incidence of VSAs also suggests that flow diverters may have a role in the management of selected patients with aneurysmal SAH. Using these devices for treatment of select VSAs can lower the risk of an intraprocedural aneurysm rupture by eliminating the need for entering the VSA with a microwire or microcatheter. ${ }^{34} 35$ The use of selfexpanding stents to treat VSAs, particularly blister aneurysms, has also been reported. ${ }^{36} 37$

The other observation to emerge from our study is the fact that, in about $10 \%$ of patients with multiple aneurysms, it is not the largest aneurysm that is responsible for rupture (figure 6). This finding also has important diagnostic and management implications. It suggests once again that risk factors other than size are important in determining the risk of rupture for intracranial aneurysms. Additionally, it also suggests that, even when risk factors related to lifestyle such as smoking and high blood pressure as well as genetic risk factors are taken into account (both the ruptured smaller aneurysms and the unruptured larger aneurysms found in each patient were subjected to the same lifestyle and genetic risk factors), specific physical hemodynamic stress factors play an important role in determining the risk for aneurysm rupture. These could include factors such as the size of the parent vessel or, in the case of aneurysms that arise at arterial origins, factors such as the angle between branches or

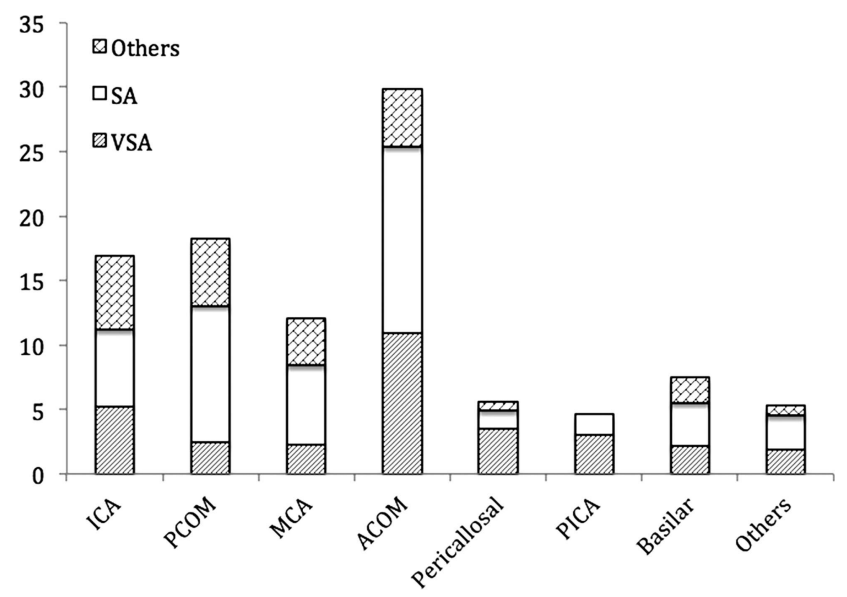

Figure 4 Incidence of ruptured aneurysms at each anatomical location. ACOM, anterior communicating artery; ICA, internal carotid artery; MCA, middle cerebral artery; PCOM, posterior communicating artery; PICA, posterior inferior cerebellar artery. differences in the relative size of arterial branches. ${ }^{38-40}$ There is an urgent need to better understand this last set of hemodynamic stress factors through focused retrospective analyses or computational modeling.

In summary, our retrospective analysis of the size and distribution of ruptured aneurysms in patients who presented to our institution with SAH over a 5 -year period showed that a significant proportion of these measured $\leq 3 \mathrm{~mm}$. Moreover, the relative incidence of ruptured aneurysms of $\leq 3 \mathrm{~mm}$ was particularly high in certain locations such as the pericallosal artery and PICA territories. We also found that, in approximately $10 \%$ of patients with multiple aneurysms who presented with SAH, the largest aneurysm was not responsible for the hemorrhage. Therefore, in patients presenting with diffuse SAH and multiple aneurysms, treatment should be considered for all the aneurysms irrespective of size, even if some are VSAs. These findings highlight the importance of improving upon the currently available prognostic, diagnostic and therapeutic tools for the management of patients who present with intracranial aneurysms.

With regard to counseling patients who present with unruptured VSAs, our results suggest that, in young patients with VSAs at specific locations such as pericallosal or PICA aneurysms, it may be reasonable to offer treatment, particularly when there is a family history of SAH or a history of smoking. Needless to say, these high-risk elective procedures are probably best performed by very experienced practitioners or their partners under their supervision.

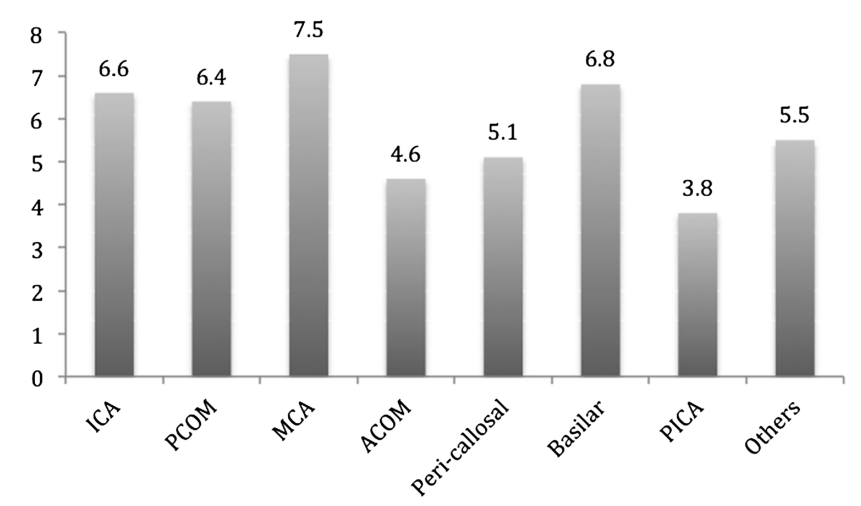

Figure 5 Mean size of ruptured intracranial aneurysms at each location in $\mathrm{mm}$. ACOM, anterior communicating artery; ICA, internal carotid artery; MCA, middle cerebral artery; PCOM, posterior communicating artery; PICA, posterior inferior cerebellar artery. 
(a)

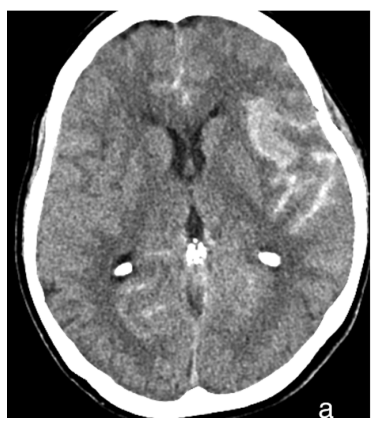

(b)

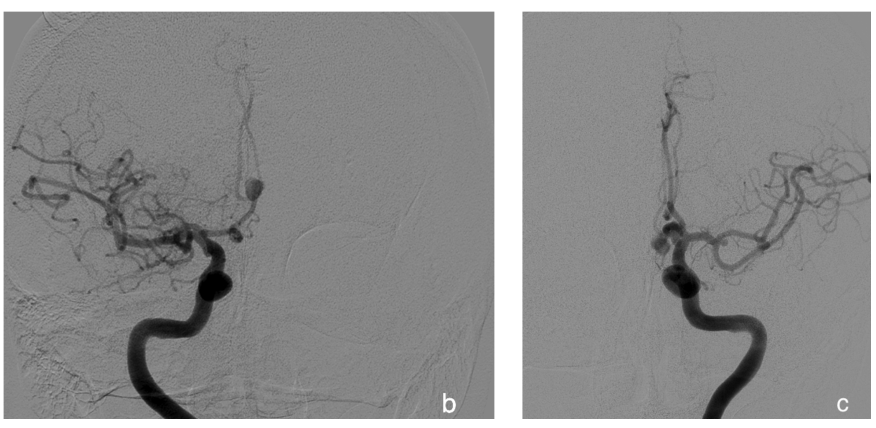

Figure 6 (A) Non-contrast head CT in a 68-year-old with subarachnoid hemorrhage (SAH) showing blood predominantly along the left sylvian fissure. (B) Digital subtraction angiography (DSA) in the anteroposterior projection following right internal carotid artery (ICA) injection in the same patient showing a $6 \mathrm{~mm}$ pericallosal aneurysm. (C) DSA in the anteroposterior projection following left ICA injection showing a $2.5 \mathrm{~mm}$ left middle cerebral artery aneurysm and a $9 \mathrm{~mm}$ anterior communicating artery aneurysm.

Contributors BDJ: Conception of the work, data collection, statistical analysis, interpretation and discussion, final approval. JEDA: Statistical analysis, interpretation and discussion, final approval. YK: Data collection, submission to Institutional Review Board, design of study, final approval. CPD, MRC, KMR, GJZ, RGD: Interpretation of data, revision for intellectual content, final approval. DTCIII: Interpretation of data, revision for critical and intellectual content, final approval. CJM: Conception of work, design of study, revision for critical and intellectual content, drafting of manuscript and final approval.

\section{Competing interests None.}

Ethics approval Ethics approval was obtained from the Institutional Review Board. Provenance and peer review Not commissioned; externally peer reviewed.

\section{REFERENCES}

1 van Gijn J, Kerr RS, Rinkel GJ. Subarachnoid hemorrhage. Lancet 2007:369:306-18

2 Wiebers DO, Whisnant JP, Huston J III, et al. Unruptured intracranial aneurysms: natural history, clinical outcome, and risks of surgical and endovascular treatment. Lancet 2003:362:103-10.

3 Forget TR Jr, Benitez R, Veznedaroglu E, et al. A review of size and location of ruptured intracranial aneurysms. Neurosurgery 2001;49:1322-6.

4 Osawa M, Hongo K, Tanaka Y, et al. Results of direct surgery for aneurysmal subarachnoid haemorrhage: outcome of 2055 patients who underwent direct aneurysm surgery and profile of ruptured intracranial aneurysms. Acta Neurochir (Wien) 2001;143:655-64.

5 Weir B, Disney L, Karrison T. Sizes of ruptured and unruptured aneurysms in relation to their sites and the ages of patients. J Neurosurg 2002;96:64-70.

6 Kassell NF, Torner JC. Size of intracranial aneurysms. Neurosurgery 1983;12:291-7.

7 Sonobe M, Yamazaki T, Yonekura M, et al. Small Unruptured Intra-cranial Aneurysm Verification Study: SUAVe Study, Japan. Stroke 2010;41:1969-77.

8 Kataoka K, Taneda M, Asai T, et al. Difference in nature of ruptured and unruptured cerebral aneurysms. Lancet 2000;355:203.

9 Wiebers DO, Whisnant JP, Sundt TM Jr, et al. The significance of unruptured intracranial saccular aneurysms. J Neurosurg 1987:66:23-9.

10 Rahman M, Ogilvy CS, Zipfel GJ, et al. Unruptured cerebral aneurysms do not shrink when they rupture: multicenter collaborative aneurysm study group. Neurosurgery 2009;917:406

11 Yonekura M. Importance of prospective studies for deciding on a therapeutic guideline for unruptured cerebral aneurysm. Acta Neurochir 2002;82(Suppl):21-2.

12 Mitchell $P$, Jakubowski J. Estimate of the maximum time interval between formation of cerebral aneurysm and rupture. J Neurol Neurosurg Psychiatry 2000;69:760-7.

13 Schievink W. Intracranial aneurysms. N Eng/ J Med 1997;336:28-40.

14 Juvela $S$. Prevalence of risk factors in spontaneous intracerebral hemorrhage and aneurysmal subarachnoid hemorrhage. Arch Neurol 1996;53:734-40.

15 Juvela $\mathrm{S}$, Hillbom $\mathrm{M}$, Numminen $\mathrm{H}$, et al. Cigarette smoking and alcohol consumption as risk factors for aneurysmal subarachnoid hemorrhage. Stroke 1993:24:639-46.

16 Longstreth WT Jr, Nelson LM, Koepsell TD, et al. Cigarette smoking, alcohol use, and subarachnoid hemorrhage. Stroke 1992;23:1242-9.

17 Nahed BV, DiLuna ML, Morgan T, et al. Hypertension, age and location predict rupture of small intracranial aneurysms. Neurosurgery 2005;57:676-83.

18 Miyazawa N, Akiyama I, Yamagata Z. Risk factors for growth of unruptured intracranial aneurysms: follow-up study by serial 0.5-T magnetic resonance angiography. Neurosurgery 2006;58:1047-53.
19 Agid R, Lee SK, Willinsky RA, et al. Acute subarachnoid hemorrhage: using 64-slice multidetector CT angiography to "triage" patients' treatment. Neuroradiology 2006;48:787-94.

20 Westerlaan HE, Gravendeel J, Fiore D, et al. Multislice CT angiography in the selection of patients with ruptured intracranial aneurysms suitable for clipping or coiling. Neuroradiology 2007;49:997-1007.

21 Lubicz B, Levivier M, François 0 , et al. Sixty-four-row multisection CT angiography for detection and evaluation of ruptured intracranial aneurysms: interobserver and intertechnique reproducibility. AJNR Am J Neuroradiol 2007;28:1949-55.

22 Westerlaan HE, van Dijk MJMC, Jansen-van der Weide MC, et al. Intracranial aneurysms in patients with subarachnoid hemorrhage: CT angiography as a primary examination tool for diagnosis — systematic review and meta-analysis. Radiology 2011:258:134-45.

23 Dammert S, Krings T, Moller-Hartmann W, et al. Detection of intracranial aneurysms with multislice $\mathrm{CT}$ : comparison with conventional angiography. Neuroradiology 2004;46:427-34

24 Tipper G, U-King-Im JM, Price SJ, et al. Detection and evaluation of intracranial aneurysms with 16-row multislice CT angiography. Clin Radiol 2005;60:565-7.

25 Yoon DY, Lim KJ, Choi CS, et al. Detection and characterization of intracrania aneurysms with 16-channel multidetector row CT angiography: a prospective comparison of volume-rendered images and digital subtraction angiography. AJNR Am J Neuroradiol 2007:28:60-7.

26 Agid R, Andersson T, Almqvist $\mathrm{H}$, et al. Negative CT angiography findings in patients with spontaneous sub arachnoid hemorrhage: when is digitial subtraction angiography still needed. Am J Neuroradiol 2010;31:696-705.

27 Hochberg AR, Rojas R, Thomas AJ, et al. Accuracy of on-call resident interpretation of $\mathrm{CT}$ angiogram for intracranial aneurysm in subarachnoid hemorrhage. Am J Roentgenol 2011;197:1436-41.

28 Nguyen TN, Raymond J, Guilbert F, et al. Association of endovascular therapy of very small ruptured aneurysms with higher rates of procedure-related rupture. J Neurosurg 2008;108:1088-92.

29 Brinjikji W, Lanzino G, Cloft HJ, et al. Endovascular treatment of very small (3 mm or smaller) intracranial aneurysms: report of a consecutive series and a meta-analysis. Stroke 2010J;41:116-21.

30 Signorelli F, Scholtes F, Bojanowski MW. Very small intracranial aneurysms: clip or coil. Neurochirurgie 2012;58:156-9.

31 Cross DT, Tirschwell D, Clark MA, et al. Mortality following subarachnoid hemorrhage varies with hospital case volume in 18 states. J Neurosurg 2003;99:810-7.

32 Leake CB, Waleed Brinjikji, Kallmes DF, et al. Increasing treatment of ruptured cerebral aneurysms at high volume centers in the United States. J Neurosurg 2011;115:1179-83.

33 Grigoryan M, Chaudhry SA, Hassan AE, et al. Neurointeventional procedural volume per hospital in the United States: implications for comprehensive stroke center designation. Stroke 2012;43:1309-14.

34 Henkes $\mathrm{H}$, Preiss $\mathrm{H}$, Miloslavski $\mathrm{E}$, et al. Endovascular treatment of small intracranial aneurysms: three alternatives to coil occlusion. Minim Invasive Neurosurg 2006;49:65-9.

35 Kulcsar Z, Wetzel SG, Augsburger L, et al. Effect of flow diversion treatment on very small ruptured aneurysms. Neurosurgery 2010;67:789-93.

36 Fiorella D, Albuqueque FC, Deshmukh VR, et al. Endovascular reconstruction with the Neuroform stent as monotherapy for the treatment of uncoilable intradural pseudoaneurysms. Neurosurgery 2006;59:291-300.

37 Bulsara KR, Kuzmik GA, Herbert R, et al. Stenting as monotherapy for uncoilable intracranial aneurysms. Neurosurgery Published Online First: 30 Nov 2012. doi:10.1227/NEU.0b013e31827fcaba. 


\section{Hemorrhagic stroke}

38 Ingebrigtsen $\mathrm{T}$, Morgan MK, Faulder $\mathrm{K}$, et al. Bifurcation geometry and the presence of cerebral artery aneurysms. J Neurosurg 2004;101:108-13.

39 Bor ASE, Velthuis BK, Majoie CB, et al. Configuration of intracranial arteries and development of aneurysms: a follow up study. Neurology 2008;70:700-5.
40 Kasuya H, Shimizu T, Nakaya K, et al. Angles between A1 and A2 segments of the anterior cerebral artery visualized by three-dimensional computed tomographic angiography and association of anterior communicating artery aneurysms. Neurosurgery 1999;45:89-93.

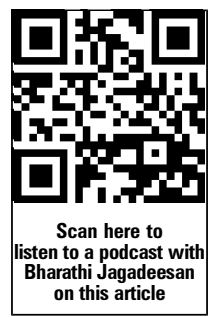

